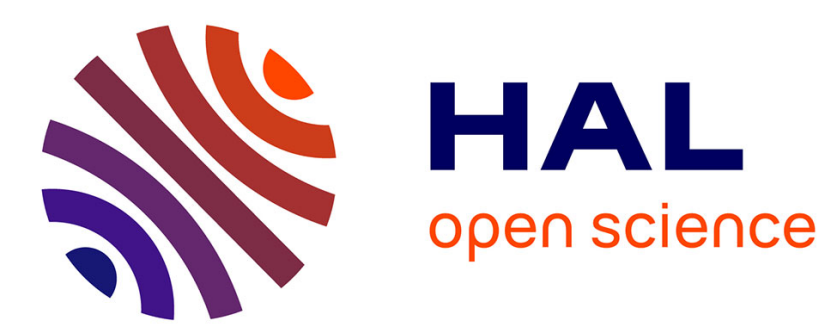

\title{
La prévention du SIDA à Bamako, une mise en contexte
}

Sébastien Tusseau, Sébastien Fleuret

\section{To cite this version:}

Sébastien Tusseau, Sébastien Fleuret. La prévention du SIDA à Bamako, une mise en contexte. Villes en parallèles, 2011, 44-45, pp.49 - 74. hal-01188088

\section{HAL Id: hal-01188088 \\ https://hal.science/hal-01188088}

Submitted on 28 Aug 2015

HAL is a multi-disciplinary open access archive for the deposit and dissemination of scientific research documents, whether they are published or not. The documents may come from teaching and research institutions in France or abroad, or from public or private research centers.
L'archive ouverte pluridisciplinaire HAL, est destinée au dépôt et à la diffusion de documents scientifiques de niveau recherche, publiés ou non, émanant des établissements d'enseignement et de recherche français ou étrangers, des laboratoires publics ou privés. 


\title{
La prévention du SIDA à Bamako, une mise en contexte
}

\author{
Sébastien Tusseau, doctorant, CARTA-ESO, Université d'Angers
}

Sébastien Fleuret, chargé de recherche CNRS, CARTA-ESO, Université d'Angers

Résumé: Les progarmmes de prévention du SIDA en Afrique sont bien souvent pensés à d'autres niveaux et omettent de tenir compte des variations mico-locales de contexte. A partir d'une enquête menée dans deux quartiers de Bamako (Mali), ce texte montre que des variations existent à l'échelle infra-urbaine dans la perception du risque face au VIH/SIDA et dans la modicication ou non des pratiques sexuelles selon le contexte et la source de diffusion des messages de prévention.

Le premier cas déclaré de SIDA au Mali date de 1985. Depuis, comme dans la majorité des pays africains, le nombre de personnes vivant avec le VIH/SIDA n'a cessé d'augmenter. Cependant, lors de la dernière Enquête Démographique de Santé au Mali (E.D.S.M. IV), le taux de prévalence du SIDA a baissé, passant de $1,7 \%$ en 2001 à 1,3\% en 2006.

$\mathrm{Si}$, dans le contexte africain, le Mali est un pays à faible prévalence et à épidémie concentrée, la problématique du SIDA n'en est pas moins cruciale et pas uniquement pour les groupes dits «à risques » que sont les routiers, les coxeurs ${ }^{1}$, les prostituées, les vendeuses ambulantes, les aides ménagères ou les enfants en rupture familiale.

L'ensemble de la population est concernée même si les tranches d'âges les plus touchées sont les plus jeunes (les $25-44$ ans pour les femmes et $30-34$ pour les hommes) et que le milieu urbain est plus infecté par le virus que les zones rurales. Bamako, la capitale, présente par exemple, un taux de prévalence de $1,9 \%$ contre $0,9 \%$ pour les zones rurales et $1,3 \%$ pour les autres villes. C'est sur cet espace urbain de Bamako, peuplé d'1,3 millions d'habitants (estimation, 2003), que porte notre étude.

Les principaux déterminants de la propagation de l'épidémie sont les comportements sexuels à risque, aggravés par l'importance du flux migratoire interne et externe, la pauvreté et les relations inégalitaires dans le genre augmentant le risque de transmission du VIH.

Nous postulons que les contextes sociaux en place sur les territoires agissent différemment sur les représentations du VIH/SIDA et donc sur la vulnérabilité des populations concernées. La diversité sociale des individus inscrite dans des territoires hétérogènes modèle les représentations de la maladie et affecte la réceptivité des messages de prévention ainsi que les comportements face au risque de contamination. De ce fait nous adoptons une démarche transdisciplinaire dans laquelle des références et emprunts à la géographie, la sociologie et l'anthropologie sont combinés. Nous nous inscrivons donc résolument dans le courant de la nouvelle géographie de la santé décrite par Kearns et Moon $(2002$; 2007) dont «le cadre de référence clé est la capacité de relier différentes perspectives théoriques (...) d'intégrer individus et lieux (...) et de faciliter la généralisation des études de cas à micro-échelle ».

Ce texte présente les résultats d'une enquête et d'une série d'entretiens menés dans deux quartiers urbains aux profils sociaux bien différenciés dans le but de mettre en évidence l'importance de facteurs contextuels micro-locaux dans l'appréhension individuelle et collective des risques, des représentations et des comportements face au VIH/SIDA.

A la lecture de ces résultats il apparaît que cette différenciation contextuelle de

\footnotetext{
${ }^{1}$ Apprentis chauffeurs vendant de tickets dans les gares
} 
représentations de la maladie est parfois en contradiction avec l'action de certains des acteurs en charge de la lutte contre le SIDA dans la mise en place de programmes de sensibilisation.

\section{Contexte}

Le Mali est un pays pauvre dont le système de santé est encore embryonnaire (Balique et al., 2001). L'économie du Mali est essentiellement basée sur l'agriculture, l'élevage et la pêche. Le Mali est classé comme pays à faible Indice de Développement Humain selon les critères du PNUD. (174ème sur 177 pays)

La population résidente du Mali est estimée à 12.3 millions d'habitants en 2006. La densité moyenne est de 9,2 habitants au $\mathrm{km} 2$. La population des zones urbaines représente $26,8 \% \mathrm{du}$ total mais la répartition spatiale est inégale avec plus de 3/4 de la population occupant moins de $10 \%$ du territoire.

Presque la moitié de la population est âgée de moins de 15 ans et la tranche d'âge 15-49 ans représente plus de $45 \%$ de l'ensemble. La religion dominante est l'islam avec environ $90 \%$ de musulmans.

La sensibilisation de la population au risque du SIDA a débuté il y a près de 10 ans maintenant au Mali mais les planificateurs d'actions de prévention ne sont pas persuadés du bien fondé de leurs actions. Cela corrobore les réflexions publiées dès la fin des années 90 par des auteurs tels que Becker (1998) ou par des organismes tels que l'OnuSIDA (1999) émettant des doutes quant à l'efficacité des messages de prévention primaire. Campbell et MacPhail pointaient en 2002 l'insuffisance des leçons tirées des succès ou insuccès des campagnes. Plusieurs responsables au Mali parlent de l'inadaptation des messages.

Un responsable malien de la cellule sectorielle de lutte contre le SIDA notait en janvier 2007 que «par rapport au cadre stratégique national, c'est toujours les mêmes idées qui apparaissent, c'est toujours le même package préventif, curatif... dans ce contexte là, les comportements ne changent pas. »

Ceci est, semble-t-il, aggravé par des problèmes de coordination entre les acteurs en charge de la lutte contre le SIDA. Pour exemple, le Dr. M., médecin au Centre de Santé Communautaire de Banconi à Bamako remarquait en janvier 2008 que les populations ne souscrivaient pas aux messages divulgués car il n'existe pas de cohérences entre les différentes sources (radio, télévision ou autre vecteur). Des acteurs sur le terrain se plaignent des difficultés à mettre en place un projet de sensibilisation à cause des exigences des bailleurs de fonds (Fonds Global et Banque mondiale). Par ailleurs les lourdeurs administratives des bailleurs freinent les efforts des associations ou O.N.G., ainsi que des responsables politiques ${ }^{2}$ l'ont fait remarquer lors de la conférence VIH/SIDA à Bamako (2007). Des auteurs tels que Mappa (2005), Schaalma (2002), Farmer (2006) ou Parkhurst et Lush (2004) notent que le transfert de modèles ou programmes occidentaux sur le territoire africain n'a pas entraîné les effets positifs escomptés dans la réponse à l'épidémie du VIH/SIDA. L'influence du modèle occidental est omniprésente à la télévision ou sur les panneaux de sensibilisation. Les logos des grandes organisations internationales sont parfois contreproductifs car ils réactivent d'anciennes croyances sur la responsabilité des occidentaux dans la propagation du SIDA.

La population malienne affiche globalement une bonne connaissance du SIDA mais les chiffres de l'Enquête Démographique de Santé au Mali (EDSM IV) montrent que les comportements évoluent difficilement. La perception du risque dépend de variables

\footnotetext{
${ }^{2}$ M. Sène, Enda Mali, Samu Social malien...
} 
contextuelles. Comme le notait en 2007, le Dr. C., médecin du Centre d'Ecoute, de Soins, d'Accueil et de Conseils (C.E.S.A.C.) à Bamako, «les problèmes de connaissances de la maladie sont avant tout un problème de scolarisation ». Nombre d'études assimilent ce risque tour à tour à la pauvreté, à la faible scolarisation. (OnuSIDA, 2008; Wagbatsoma \& Okojie, 2006, Asfhorth, 2002) Les conclusions du travail de C. Boileau (2007) invitent également à travailler sur le genre, les conditions sociales (facteurs contextuels) et les facteurs relationnels (famille).

Ce que nous observons, ce sont des épidémies différentes selon les pays, les régions, les quartiers. Epidémies qui ont chacune leurs caractéristiques et leurs contextes, c'est pour cela que des sensibilisations formatées ne fonctionnent pas partout. Pour preuve, les réussites de l'Ouganda et de Thaïlande ne sont pas liées aux mêmes modes de prévention. Le Mali est depuis des siècles un carrefour pour les ethnies ou les populations, les quartiers, les villes, les régions sont des mosaïques d'ethnies, de religions, de cultures... Nos entretiens exploratoires montrent que, sur le terrain, les acteurs de la prévention sont convaincus :

- «qu'il est nécessaire de prendre en compte le contexte pour tester les messages, les élaborer » (Un responsable de l'USAid)

- «qu'il est regrettable que l'écologie, le milieu ne soient pas pris en compte»(O.N.G. GIMPDS $^{3}$ )

A partir de ce constat, nous avons élaboré une méthodologie d'étude qui réponde aux enjeux identifiés : prendre en compte le contexte géographique à l'échelle micro-locale dans l'étude de la réception des messages de prévention par la population dans son ensemble.

\section{Méthode}

Il ne faut pas s'attacher à évaluer la prévention uniquement au regard de la courbe de prévalence ou des connaissances de messages généraux, mais sur la modification de tout un ensemble de comportements.

Connaître les caractéristiques locales de l'épidémie est un élément essentiel pour que les efforts de prévention soient efficaces (PIOT, 2008) cela suppose de mettre en place des outils d'enquête quantitative, des entretiens qualitatifs et une approche de terrain eux-mêmes adaptés au contexte.

Les résultats que nous présentons plus loin dans ce texte reposent donc sur une méthodologie originale qui associe une enquête générale de population et des entretiens avec les acteurs de la santé.

\section{- L'enquête}

L'enquête a été conduite en deux phases, l'une en janvier-février 2008, l'autre en septembredécembre 2008 auprès d'un échantillon représentatif de la population $(\mathrm{n}=962)$ dans deux quartiers de Bamako choisis pour leurs profils (contextes) socio-spatiaux différenciés.

Bamako, capitale du Mali, est au carrefour et à la convergence des principales voies routières du pays. Ces routes qui traversent Bamako de part en part marquent d'ailleurs fortement la structure de l'urbanisation comme en témoigne la configuration stellaire de la ville (Carte 1) La diversité de la population bamakoise est liée à l'histoire de la capitale qui n'a cessé d'être un point de rencontre entre des groupes d'origines diverses. La ville est toujours en croissance, environ 17000 personnes affluent chaque année. Si les quartiers du village de

\footnotetext{
${ }^{3}$ Groupement d'Initiatives Maliennes Pour le Développement Social
} 
Bamako abritaient lors de la pénétration coloniale des communautés appartenant à des lignages distincts, cela n'est plus le cas dans le Bamako actuel. Il résulte du mode d'attribution indifférencié des parcelles depuis l'administration coloniale, une "hétérogénéité de la population des quartiers de Bamako (qui) s'oppose à l'homogénéité ethnique, voire clanique ou lignagère du village. » (SINOU A. 1986)

Par ailleurs, la ville est coupée en deux par le fleuve Niger et présente une forte différenciation entre la rive gauche et la rive droite tant en termes démographiques que fonctionnels. Sur la rive gauche, site de l'ancien centre colonial sont implantés des quartiers d'urbanisation ancienne. 4 communes sur les 6 composants le district de Bamako se situent sur cette rive, cela représente plus de $70 \%$ de la population de Bamako.

Pour notre enquête, nous avons choisi de travailler dans deux quartiers : Banconi et Faladié (carte 1). Ce choix n'est pas formel. En effet, il est particulièrement compliqué à Bamako, d'accéder à des données fiables et recueillies systématiquement dans un cadre spatialisé. Dès lors il ne nous a pas été possible de construire une typologie socio-spatiale ${ }^{4}$ ni d'utiliser de façon systématique une base statistique comme «témoin » ou «étalon ». Néanmoins nous avons porté une attention toute particulière à ce que les deux terrains choisis à titre d'exemples présentent des caractéristiques comparables à la fois similaires (quartiers périphériques, situés sur des axes de sortie de ville) et opposables (du point de vue des niveaux de vie, du type d'habitat, de la confession dominante notamment).

Banconi se situe en commune 1 du district de Bamako, sur la rive gauche du fleuve Niger. C'est un quartier périphérique de la ville composé pour partie d'habitat ancien et qui, par ailleurs, gagne du terrain sur les collines de Bamako par l'implantation d'un habitat spontané. Cette extension est en grande partie liée à un exode rural important et à un fort taux d'accroissement naturel. La partie plus ancienne de Banconi a conservé son caractère populaire avec un centre de santé communautaire efficace. Le quartier est multi-ethnique mais avec une population en grande partie de confession musulmane.

Faladié est en commune 6 du district de Bamako, sur la rive droite du Niger. Il s'agit aussi d'un quartier périphérique de la ville sur la route très fréquentée de Sikasso, zone aurifère et cotonnière. Faladié est un quartier d'habitat résidentiel aisé. Ce quartier est caractérisé par une plus grande proportion de population de confession chrétienne.

Dans ces deux quartiers, la passation de l'enquête a été effectuée par des étudiants du département de géographie de la faculté des lettres et sciences humaines de l'Université de Bamako ce qui a permis d'interroger des personnes maîtrisant mal le français en traduisant les questions en Bambara.

\footnotetext{
${ }^{4}$ Sur la base d'une Analyse en composantes principales doublée d'une classification ascendante hiérarchique par exemple.
} 
Cartes 1 et 2 : Terrains d'étude à Bamako phases d'urbanisation et profils d'habitat.
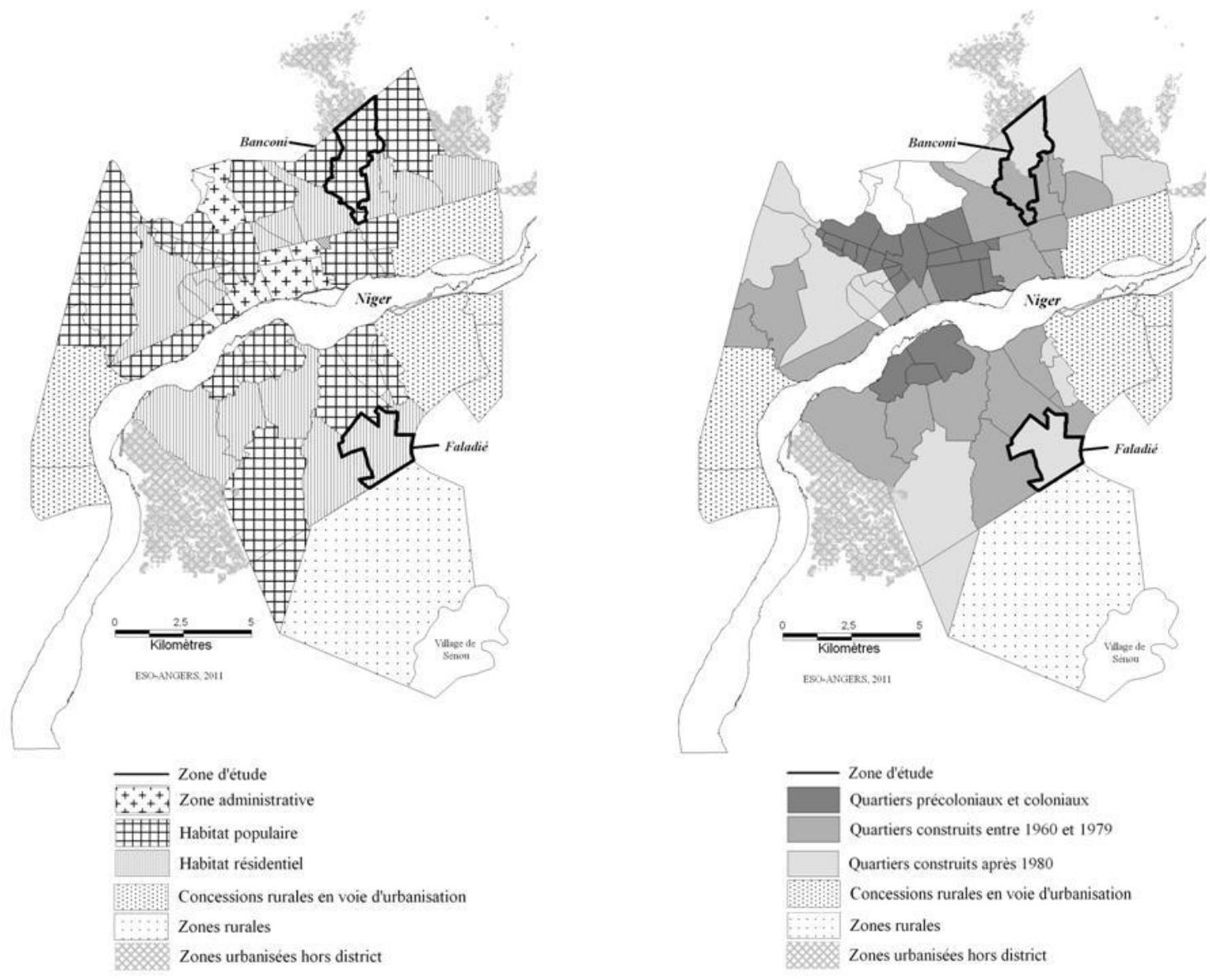

Source : I.G.M

La différenciation entre ces deux quartiers-exemples apparaît, et vient justifier leur choix, lorsque l'on réalise le test du Chi2 sur la variable «connaissance du VIH/Sida à partir des résultats de l'enquête (tableau 1)

Tableau 1 : Dépendance de la variable « connaissance du VIH/SIDA au lieu d'habitation »

\begin{tabular}{|c|c|c|c|}
\hline $\begin{array}{l}\begin{array}{r}\text { Connaissance du } \\
\text { VIH/SIDA }\end{array} \\
\text { Lieu d'habitation }\end{array}$ & $\begin{array}{l}\text { Oui } \\
(\%)\end{array}$ & $\begin{array}{l}\text { Non } \\
(\%)\end{array}$ & $\begin{array}{l}\text { La dépendance au lieu d'habitation est très } \\
\text { significative : } \\
\text { chi2 }=29,34 ; \mathrm{ddl}=1 ; \mathrm{p}=0,01\end{array}$ \\
\hline Banconi & 75.3 & 24.7 & \\
\hline Faladié & 95.5 & 4.5 & Les cases grisées sont celles pour lesquelles \\
\hline Total & 85.3 & 14.7 & $\begin{array}{l}\text { l'effectif réel est nettement supérieur/inférieur } \\
\text { à l'effectif théorique. }\end{array}$ \\
\hline
\end{tabular}

Les grandes parties de l'enquête étaient composées de questions relatives à l'état civil, au capital social (réseau familial et amical, membre d'une association), à l'accès aux soins (choix du praticien, raisons de ce choix), à l'accès aux différentes sources d'informations, aux connaissances sur le SIDA, aux comportements sexuels et comportements vis-à-vis du SIDA, à l'utilisation du préservatif et aux opinions sur les actions de prévention et sur les différents acteurs de cette prévention. 
Les résultats que nous présentons dans ce texte reposent sur une exploitation partielle de cette enquête: le dépouillement de 362 questionnaires (181 pour chacun des quartiers) correspondant à la phase 1 de ce travail.

\section{- $\quad$ Les entretiens}

L'enquête a été complétée par une série d'entretiens auprès d'acteurs de santé et d'acteurs spécialisés dans le domaine de la prévention du SIDA au Mali qu'ils soient de statut associatif, non-gouvernemental, institutionnel ou international.

La lutte contre le SIDA au Mali est politiquement gérée par le Haut Conseil National de Lutte contre le SIDA sous l'égide du Président de la République lui-même. Cette structure récolte les fonds des principaux bailleurs de fonds qui, au Mali sont, la Banque Mondiale par le biais du programme M.A.P. et le Fond Global.

En 2006, le financement extérieur demeurait la principale source de financement de la lutte contre le VIH/SIDA et les IST au Mali avec 74\% des ressources et dépenses totales.

Figure 1.

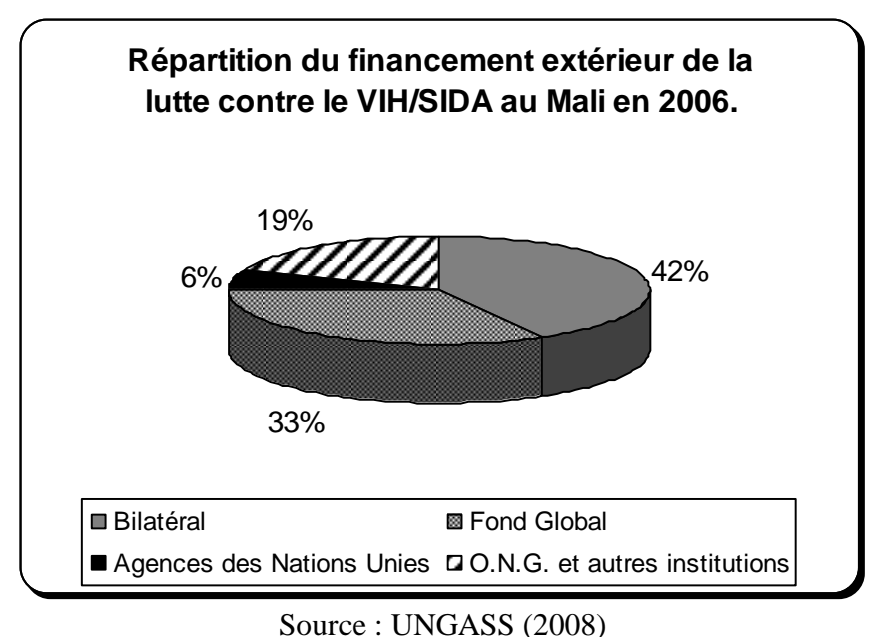

Source : UNGASS (2008)

Au total, 31 entretiens ont été réalisés sur une période allant de janvier 2007 à octobre 2008 (tableau 1). Ces entretiens ont été conduits selon une grille semi-directive portant sur la conception des programmes de prévention du SIDA, la prise en compte du contexte dans lequel ou lesquels les acteurs rencontrés sont implantés, les populations ciblées, les techniques de diffusion des messages de prévention, les réseaux et collaborations entre les acteurs de statut différent (associatif, politique, international...). 
Tableau 2 : les personnes rencontrées selon leur statut et leur fonction.

\begin{tabular}{|l|l|}
\hline \multicolumn{1}{|c|}{ Statut } & \multicolumn{1}{c|}{ Fonctions des personnes rencontrées } \\
\hline $\begin{array}{l}\text { Institutions maliennes } \\
\text { (3 entretiens) }\end{array}$ & $\begin{array}{l}\text { Responsable de programme du Haut conseil national de lutte contre le } \\
\text { SIDA; Responsable de la cellule sectorielle de lutte contre le SIDA } \\
\text { (Ministère de la santé) ; Président de l'alliance des religieux du Mali }\end{array}$ \\
\hline $\begin{array}{l}\text { Organisations internationales } \\
\text { 6 entretiens) }\end{array}$ & $\begin{array}{l}\text { Responsable de programme du Center Disease Control (USA); } \\
\text { Président et responsable de programme de l'USAID; Responsable de la } \\
\text { coopération française au sein de la cellule sectorielle de lutte contre le } \\
\text { SIDA; Président de l'ONUSIDA à Bamako; Responsable de } \\
\text { programme à la Banque Mondiale }\end{array}$ \\
\hline $\begin{array}{l}\text { Associations et ONG } \\
(18 \text { entretiens) }\end{array}$ & 8 responsables de programmes ; 10 présidents d'ONG ou associations \\
\hline $\begin{array}{l}\text { Professionnels de santé } \\
(4 \text { entretiens) }\end{array}$ & 2 responsables de CSCOM ${ }^{6}, 2$ responsables d'ASACO ${ }^{7}$ \\
\hline
\end{tabular}

\section{Résultats}

$\mathrm{Au}$ Mali, la pratique de l'IEC (Information Education Communication) est l'un des axes essentiels de la prévention du VIH et du SIDA. Elle consiste en la promotion de plusieurs messages notamment : l'abstinence sexuelle, la fidélité, la limitation du nombre de partenaires sexuels, l'usage régulier du préservatif, l'adoption des comportements sexuels à moindre risque. Les résultats de notre étude révèlent des différences de niveaux d'information, de perceptions et de représentations selon le contexte géographique et démontrent les limites des actions de prévention en pointant deux carences :

- Un manque d'accès à l'information ou un mauvais ciblage de l'information qui ont pour conséquence une faible modification des comportements à risque.

- Une relative absence de cohérence territoriale entre les différents acteurs qui a pour conséquence un enchevêtrement de discours parfois contradictoires ou contre-productifs.

\section{- Des perceptions différenciées}

Comme mentionné précédemment dans la présentation des terrains retenus, nous avons recherché dans les premiers résultats de l'enquête quantitative, des éléments de différenciation entre les quartiers de Banconi et de Faladié. La première différence notable est dans le degré de connaissance même de la maladie (Tableau 1). La population du quartier plus aisé de Faladié déclare à 95.6\% avoir connaissance de l'existence de la maladie alors qu'un quart de la population de Banconi déclare ne pas connaître le SIDA.

Au-delà de la conscience de l'existence de la maladie, des différences notables apparaissent dans le niveau d'information et de savoirs à son sujet. La variation de la part des habitants de chaque quartier étudié affichant une bonne connaissance des modes de transmission du virus en est une bonne illustration. Les résidents de Faladié affichent un taux systématiquement supérieur d'au moins 10\% à ceux de Banconi pour les modes de transmission que sont les rapports sexuels, la transfusion sanguine, les objets coupants souillés ou la seringue. Cet écart augmente jusqu'à $20.9 \%$ au sujet de la transmission mère-enfant.

\footnotetext{
${ }^{5}$ Liste des associations et ONG rencontrées : Enda Mali ; Jeunesse et développement ; Musowjigi ; Djekafo ; Jigi ; PSI Mali ; Action santé ; Stop SIDA ; Save the children Canada ; Soutoura ; Co-GIMPDS ; Amifa ; Adera 24 ; Groupe pivot-santé-population ; CAFO ; Care Mali ; CERDEPE ; SAMU social Malien

${ }^{6}$ CSCOM : Centre de Santé Communautaire

${ }^{7}$ ASACO : Association de Santé Communautaire
} 
Tableau 3 : Connaissance des modes de transmission du virus

\begin{tabular}{|c|c|c|}
\hline $\begin{array}{c}\text { D'après vous, quel (s) est (sont) le } \\
\text { (s) mode (s) de transmission du } \\
\text { VIH/SIDA? }\end{array}$ & Banconi & Faladié \\
\hline Sexe & $71,3 \%$ & $80,7 \%$ \\
\hline Transfusion sanguine & $65,2 \%$ & $79,6 \%$ \\
\hline Objets coupants souillés & $53,6 \%$ & $65,2 \%$ \\
\hline Transmission mère-enfant & $34,3 \%$ & $55,2 \%$ \\
\hline Seringue & $54,7 \%$ & $62,4 \%$ \\
\hline Préservatif* & $4,4 \%$ & $11,6 \%$ \\
\hline Sorcellerie & $0,6 \%$ & $3,3 \%$ \\
\hline Salive & $1,7 \%$ & $1,7 \%$ \\
\hline Ne sait pas & $21,5 \%$ & $10,5 \%$ \\
\hline
\end{tabular}

* Il existe une croyance au Mali qui postule que le lubrifiant qui recouvre les préservatifs serait un vecteur du SIDA.

Si les habitants de Faladié semblent mieux ou plus informés, ils semblent également plus perméables aux croyances et à la rumeur. Ainsi 11.6\% attribuent la contamination par le SIDA à l'usage des préservatifs contre seulement $4.4 \%$ à Banconi. Une autre question de l'enquête visait à évaluer la rationalité des personnes interrogées dans leur appréhension de la maladie. Quand il leur était demandé s'ils avaient une idée de l'origine du VIH SIDA, si une majorité attribuent la maladie à l'évolution des pratiques sexuelles (avec une différence : $50.3 \%$ à Banconi contre $68 \%$ à Faladié), 37\% des habitants de Faladié pensent qu'il s'agit d'une invention des «blancs » (ils sont seulement $12.7 \%$ à Banconi) et $34 \%$ pensent que la responsabilité en incombe à Dieu (18.2\% à Banconi).

Il semble donc que le niveau plus élevé de connaissance constaté à Faladié soit trompeur et que les croyances et informations fallacieuses s'entremêlent.

Une explication au fait que la population de Faladié est beaucoup mieux informée que celle de Banconi réside dans le constat d'un plus grand nombre d'interlocuteurs. Or, cette information plus conséquente par rapport à Banconi peut également entraîner un sentiment de «surinformation » en mettant en avant le caractère excessif de cette sensibilisation. Ainsi la c'est paradoxalement à Faladié, où le SIDA est le plus largement considéré comme une maladie mortelle, que le sentiment d'une exagération des dangers du SIDA est le plus fort .(Tableau 4). La croyance assimilant le SIDA au paludisme transmis par le biais des piqûres de moustiques est également répandue mais de façon assez similaire dans les deux quartiers.

Tableau 4 : Opinion sur le VHI/SIDA

\begin{tabular}{|c|c|c|}
\hline Le VIH/SIDA est-il : & Banconi & Faladié \\
\hline Mortel & $49,2 \%$ & $65,7 \%$ \\
\hline Paludisme aggravé & $22,1 \%$ & $27,1 \%$ \\
\hline Mensonge & $18,8 \%$ & $16,6 \%$ \\
\hline Excessif & $16,6 \%$ & $32 \%$ \\
\hline Pas grave & $13,8 \%$ & $16,6 \%$ \\
\hline Sans opinion & $19,3 \%$ & $11,6 \%$ \\
\hline
\end{tabular}

Sur le sujet du VIH/SIDA, la population de Banconi dispose de moins d'interlocuteurs par rapport aux habitants de Faladié et ce, quels que soient les canaux d'informations considérés, qu'ils soient interpersonnels (pairs éducateurs, agents de santé, école), personnels (famille, conjoint -e, amis) ou de médias de masse (à l'exception notable de la radio). 
Tableau 5 : Sources d'informations et de connaissances sur le SIDA

\begin{tabular}{|c|c|c|}
\hline $\begin{array}{c}\text { Qui vous a transmis vos } \\
\text { connaissances sur le SIDA ? }\end{array}$ & Banconi & Faladié \\
\hline Ecole & $23,8 \%$ & $44,2 \%$ \\
\hline Pairs éducateurs & $10,5 \%$ & $18,8 \%$ \\
\hline Agents de santé & $22,1 \%$ & $32,6 \%$ \\
\hline Campagnes de masse & $18,2 \%$ & $29,3 \%$ \\
\hline Acquises par moi-même & $8,3 \%$ & $25,4 \%$ \\
\hline Mon (ma) conjoint $(e)$ & $2,2 \%$ & $14,4 \%$ \\
\hline Famille & $7,2 \%$ & $26,5 \%$ \\
\hline Amis & $29,3 \%$ & $55,2 \%$ \\
\hline Radio & $65,2 \%$ & $63,5 \%$ \\
\hline Télé & $60,8 \%$ & $70,2 \%$ \\
\hline Presse & $17,7 \%$ & $21 \%$ \\
\hline Rumeur & $23,2 \%$ & $27,6 \%$ \\
\hline Autres & $3,9 \%$ & $3,3 \%$ \\
\hline
\end{tabular}

Les écarts entre niveaux d'information semblent se retrouver dans les perceptions individuelles du risque VIH./SIDA. Ainsi la population du quartier de Banconi sous-estime plus sa vulnérabilité que celle de Faladié (Tableau 6). On retrouve dans ce tableau une tendance observée dans le tableau 4 montrant que seuls $49.2 \%$ des résidents de Banconi attribuent un caractère mortel au SIDA contre $65.7 \%$ de ceux de Faladié.

Tableau 6 : Perception du risque VIH/SIDA

\begin{tabular}{|c|c|c|}
\hline $\begin{array}{c}\text { Quel est votre sentiment de } \\
\text { vulnérabilité vis-⿳亠丷⿵冂-vis } \boldsymbol{d u} \\
\text { VIH/SIDA ? }\end{array}$ & Banconi & Faladié \\
\hline Vulnérable & & \\
\hline Peu vulnérable & $27,6 \%$ & $35,4 \%$ \\
\hline Pas du tout vulnérable & $24,3 \%$ & $34,8 \%$ \\
\hline Ne sait pas & $43,1 \%$ & $23,2 \%$ \\
\hline
\end{tabular}

Les niveaux d'information différents sont un élément à prendre en considération et à associer à d'autres éléments contextuels pour analyser les représentations sociales de la maladie. Il existe par exemple beaucoup plus de tabous sur le VIH/SIDA dans le quartier populaire de Banconi. Les personnes vivant avec le VIH/SIDA y sont plus stigmatisées (Tableau 7). Le sentiment de gêne vis-à-vis de la sexualité et du SIDA apparaît à la passation de l'enquête et se traduit notablement dans certaines questions. (Tableau 8)

Tableau 7 : Le tabou du SIDA

\begin{tabular}{|c|c|c|}
\hline $\begin{array}{c}\text { Si une personne apprend qu'elle } \\
\text { est infectée par le VIH/SIDA, } \\
\text { doit-elle être autorisée à garder le } \\
\text { secret ou l'information doit-elle } \\
\text { être divulguée à la population? }\end{array}$ & Banconi & \\
\hline Secret & & \\
\hline Informer & $43,3 \%$ & $17,7 \%$ \\
\hline
\end{tabular}


Tableau 8 : Les interlocuteurs sur le SIDA face aux tabous.

\begin{tabular}{|c|c|c|}
\hline $\begin{array}{c}\text { De qui accepteriez-vous recevoir } \\
\text { des conseils ou des } \\
\text { recommandations sur la sexualité } \\
\text { en générale et sur le SIDA en } \\
\text { particulier? }\end{array}$ & Banconi & Faladié \\
\hline Médecins (hopital, cscom ...) & $60,2 \%$ & $58,6 \%$ \\
\hline Médecin traditionnel & $14,9 \%$ & $25,4 \%$ \\
\hline Autorités & $32 \%$ & $44,8 \%$ \\
\hline Amis & $65,7 \%$ & $69,6 \%$ \\
\hline Famille & $26,5 \%$ & $47 \%$ \\
\hline Membres de la communauté & $13,8 \%$ & $40,9 \%$ \\
\hline ONG & $26,5 \%$ & $49,7 \%$ \\
\hline Religieux & $30,9 \%$ & $40,3 \%$ \\
\hline Pairs éducateurs & $25,4 \%$ & $40,3 \%$ \\
\hline Médias & $40,3 \%$ & $48,6 \%$ \\
\hline Autres & $7,7 \%$ & $16 \%$ \\
\hline
\end{tabular}

La gêne exprimée par la population de Banconi s'observe par la place des interlocuteurs appartenant au quartier: les autorités, le médecin traditionnel, la famille, les amis, les religieux ou les pairs éducateurs moins considérés comme interlocuteurs acceptables sur le sujet du SIDA qu'ils ne peuvent l'être à Faladié.

Le nombre d'interlocuteurs potentiels est également plus faible à Banconi ce qui explique que les habitants y ont moins accès à l'information. Or ce manque n'est pas comblé par une plus grande communication au sein de la communauté ou par des initiatives individuelles. Peu nombreux sont ceux en effet qui citent les conjoints, les familles, les amis, les pairs éducateurs, les agents de santé ou l'école comme sources de connaissances (Tableau 5). Et seuls 8,3\% des habitants de Banconi ayant des connaissances sur le VIH/SIDA sont allés volontairement se renseigner sur la maladie contre plus de 25\% pour Faladié.

D'autres facteurs de différenciation sont à prendre en compte. Certaines variables montrent par exemple que la distinction de genre est aggravée localement (Tableaux 9 et 10).

Tableau 9: D'après vous, une personne qui a l'air en bonne santé peut-elle être atteinte du VIH/SIDA ?

\begin{tabular}{|c|c|c|c|}
\hline Banconi & Oui & Non & Ne sait pas \\
\hline Hommes & $60,8 \%$ & $15,2 \%$ & $24 \%$ \\
\hline Femmes & $42,9 \%$ & $12,5 \%$ & $44,6 \%$ \\
\hline Faladié & Oui & Non & Ne sait pas \\
\hline Hommes & $71,7 \%$ & $5,8 \%$ & $22,5 \%$ \\
\hline Femmes & $71,7 \%$ & $3,3 \%$ & $25 \%$ \\
\hline
\end{tabular}

Au-delà de la différence interquartiers déjà observée au prisme d'autres variables, si à Faladié hommes et femmes montrent le même niveau de connaissance sur l'existence de porteurs sains, à Banconi les femmes sont nettement moins bien informées que les hommes. Cela renvoie au niveau d'éducation auquel elles ont accès et plus largement à leur place dans la communauté locale.

La distinction de genre apparaît également très nettement dans les comportements face à la maladie. Ainsi l'usage du préservatif (Tableau 9) est moins répandu parmi les femmes que 
parmi les hommes et cette différence est accentuée dans le quartier de Banconi. Cette variabilité géographique ne semble pas devoir être attribuée à un écart dans les niveaux de connaissance puisque selon notre enquête, les femmes sont aussi nombreuses que les hommes à assimiler le préservatif masculin et féminin à un mode de protection vis-à-vis du VIH/SIDA et ce, dans les deux quartiers en question. Il s'agit probablement plus, dans ce cas, de conventions sociales. Celles-ci se retrouvent dans d'autres questions posées par l'enquête. Au fait de savoir si il est correct d'avoir toujours un préservatif sur soi, les réponses affirmatives sont deux fois moins fréquentes pour les femmes que pour les hommes, quel que soit l'espace considéré.

Tableau 10 : Avez-vous déjà utilisé un préservatif ?

\begin{tabular}{|c|c|c|c|}
\hline Banconi & Oui & Non & $\begin{array}{c}\text { Ne souhaite } \\
\text { pas répondre }\end{array}$ \\
\hline Hommes & $51,2 \%$ & $42,4 \%$ & $6,4 \%$ \\
\hline Femmes & $37,5 \%$ & $57,1 \%$ & $5,4 \%$ \\
\hline
\end{tabular}

\begin{tabular}{|c|c|c|c|}
\hline Faladié & Oui & Non & $\begin{array}{c}\text { Ne souhaite } \\
\text { pas répondre }\end{array}$ \\
\hline Hommes & $74,2 \%$ & $16,7 \%$ & $9,1 \%$ \\
\hline Femmes & $56,7 \%$ & $26,7 \%$ & $16,6 \%$ \\
\hline
\end{tabular}

\section{- L'enjeu de la diffusion des messages}

Dès lors que l'on prend en compte l'hétérogénéité des publics vis-à-vis desquels un discours et des actions de prévention doivent être mis en place, il apparaît évident que les messages doivent être multiformes. Or les résultats de l'enquête bamakoise ainsi que le contenu des entretiens démontrent que les messages de prévention ${ }^{8}$ passent mal.

A partir de nos enquêtes, tout comme dans la littérature internationale, nous relevons que des effets inattendus consécutifs à une information préventive trop abondante ou trop ciblée peuvent avoir des effets pervers. Dans certains cas, l'information peut susciter un certain fatalisme, un relativisme vis-à-vis des risques sanitaires. Dans d'autres cas, l'information préventive peut alimenter des réactions discriminatoires à l'égard de groupes à risque, ce qui pose déjà un problème en soi, mais ce qui peut aussi se traduire par un déni du risque.

Dans d'autre cas encore c'est la source d'où émane le message ainsi que sa forme qui peuvent s'avérer problématiques. Or cet aspect comporte une dimension géographique car la majorité des messages de prévention sur le SIDA au Mali sont importés par les bailleurs de fonds comme la Banque Mondiale qui, par exemple, oblige certains acteurs de programmes à insérer des messages pour les prostituées bien qu'il n'y ait pas de prostitution sur leur terrain de travail (Mme L., O.N.G. Samu Social Malien, 01/2007).

Un autre aspect géographique est la non prise en compte des réalités locales. Les grandes institutions qui délivrent de multiples messages le font régulièrement en français et sur support écrit. Or, environ $30 \%$ de la population malienne est analphabète et s'il n'existe pas de données précises sur les langues parlées au Mali, notre travail de terrain nous a permis de constater qu'une part importante de la population est plus à l'aise pour s'exprimer en bambara qu'en français. Ainsi seuls 13,3 \% des enquêtés de Banconi connaissent le slogan de lutte

\footnotetext{
${ }^{8}$ Nous avons essentiellement examiné les messages de prévention primaire, c'est-à-dire celle qui consiste à anticiper des phénomènes risquant d'entraîner ou d'aggraver des problèmes de santé.
} 
contre le SIDA au Mali (i.e. abstinence, fidélité, préservatif formulé en français) et 23,9\% pour Faladié.

«Les messages sont à l'avance élaborés et appliqués sur le terrain sans soucis d'adaptation. Il arrive souvent que le contenu du message (...) s'attaque à un problème particulier n'existant pas au sein de la communauté donnée. De même, certaines notions comme le plaisir sont secondaires où le lieu de l'I.E.C. et les cibles choisies ne sont pas appropriés. » (TRAORE L.T., 2006)

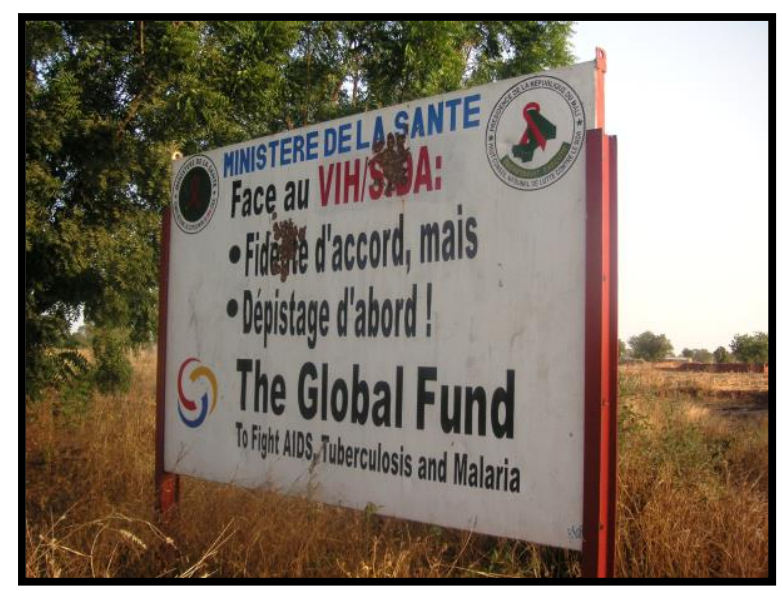

Figure 2 :

Les programmes mettent en avant le «logo» du financeur. Photo prise sur la route reliant

Bamako à l'aéroport de Bamako-Sénou (27/01/2008).

La visibilité du mécène comporte des effets pervers car elle s'avère parfois contre-productive. En effet, une part non négligeable de la population continue de croire que le SIDA «est une maladie inventée par les «blancs» ( (voir précédemment) et n'ont qu'une confiance limitée dans les organisations internationales. Le quartier populaire de Banconi est plus affecté par ce phénomène que celui de Faladié. Or la présence sur les supports d'information de logos des grandes organisations occidentales a pour effet de renforcer cette croyance. L'argument développé est que "les blancs sous couvert de campagne de prévention font la publicité pour leurs médicaments»

La prise en compte du contexte dans lequel les messages de sensibilisation sont divulgués est une des conditions à leur réussite. Les acteurs rencontrés sur le terrain regrettent que les messages de prévention se consacrent encore à la sensibilisation et non aux changements de comportements, prolongement logique de la première phase de sensibilisation. (M. T., O.N.G. CERDEPE, entretien 30/01/07, M. A., C.D.C, entretien 6/02/2007)

De fait, les programmes de lutte contre le SIDA au Mali traduisent une concentration sur le curatif. Le Mali est un des seuls pays africain avec un taux de couverture d'environ $50 \%$ du traitement pour les personnes vivant avec le VIH/SIDA ce qui est présenté politiquement comme une fierté nationale. Le revers de la médaille apparaît dans les résultats de la dernière enquête de santé qui ont révélé une baisse relative du taux de prévalence du VIH/SIDA provoquant un certain «désintérêt » envers la prévention primaire, certains considérant que les populations sont désormais suffisamment informées. Or, notre enquête démontre que cela n'est pas vrai pour l'ensemble du territoire (cf tableau 2). L'enquête nationale de santé (2006) abonde dans ce sens également. En effet, si au plan national $86 \%$ des femmes et $91 \%$ des hommes connaissent l'existence du SIDA, seulement $19,5 \%$ des femmes et $28,8 \%$ des hommes ont une connaissance complète $^{9}$ du VIH/SIDA. De plus, les niveaux de

\footnotetext{
${ }^{9}$ Sont considérés comme ayant une connaissance complète, les hommes et femmes qui déclarent que l'on peut réduire le risque de contracter le virus du SIDA en utilisant des condoms et en limitant les rapports sexuels à un seul partenaire fidèle et qui n'est pas infecté, qui rejettent les idées erronées les plus courantes à propos de la
} 
connaissance du SIDA sont en légère baisse entre les deux dernières enquêtes de santé (2001 et 2006), les chiffres pour 2001 étant de $90 \%$ pour les femmes et de $98 \%$ pour les hommes.

Enfin, «il existe un manque de recherches au préalable. Il n'existe pas d'état des lieux, le travail débute directement en phase II c'est-à-dire en phase opérationnelle » (M. T., O.N.G. MUSOWJIGI, entretien 2/10/2008). Ce manque est également exprimé par les acteurs de terrain, par les responsables politiques et associatifs. (M. B., ONG ENDA Mali, entretien 29/09/2008)

Lors des $4^{\text {èmes }}$ journées de conférence sur le VIH/SIDA de Bamako de janvier 2007, les responsables politiques maliens en charge de la lutte contre le SIDA ont fait état de leur besoin de recherches scientifiques sur les programmes de prévention et notamment sur leur évaluation. De plus, un forum ministériel mondial sur la recherche pour la santé s'est déroulé à Bamako les 17, 18 et 19 novembre 2008 avec pour objectif, «un appel à l'action de Bamako sur la recherche pour la santé. »

$\mathrm{Au}$ Mali, 37\% des ressources ont été allouées aux programmes de prévention, $23 \%$ à la gestion et coordination des programmes, $19 \%$ au traitement et soins, $15 \%$ aux ressources humaines et $3 \%$ des ressources au soutien aux orphelins et enfants vulnérables. Les autres postes de dépense (protection sociale et services sociaux, environnement favorable et développement communautaire et recherche) n'ont bénéficié que de $3 \%$ des financements au titre de l'année 2006.

Le manque de contextualisation principale carence de connaissances à l'amont de l'élaboration des messages de prévention entraîne leur inadaptation. Dans une société africaine empreinte de coutumes, de traditions, il est difficile d'intégrer des connaissances et des pratiques nouvelles, celles-ci remettant en jeu l'équilibre pré-existant.

\section{- L'enjeu de la cohérence territoriale des acteurs}

S'il est une réussite à souligner par rapport à d'autres pays africains, c'est dans l'accès important aux AntiRétroViraux (ARV), le Mali faisant partie d'un groupe restreint de pays permettant l'accès aux traitements à plus de 50\% des personnes vivant avec le VIH.

Cet accès à la prise en charge est une réussite. Mais au-delà du curatif, le préventif demeure un enjeu. Selon le directeur du Programme mondial de l'OMS intitulé Global Program on Evidence for Health Policy, "nous devons parvenir à un meilleur équilibre à l'échelle mondiale entre la prévention des maladies et le simple traitement de leurs conséquences. » Or, pour M. V., membre de la Cellule Sectorielle de la Lutte contre le SIDA au sein du Ministère de la Santé, "il y a 10 ans, c'était le tout préventif, de nos jours, c'est le tout curatif.» (M. V., C.S.L.S., entretien 29/01/2007)

Outre un manque de considération et de moyens dans les dispositifs nationaux, la prévention primaire souffre du manque de coordination des acteurs sur le terrain. 
Figure 3. Les patients bénéficiaires d'AntiRétroViraux au Mali

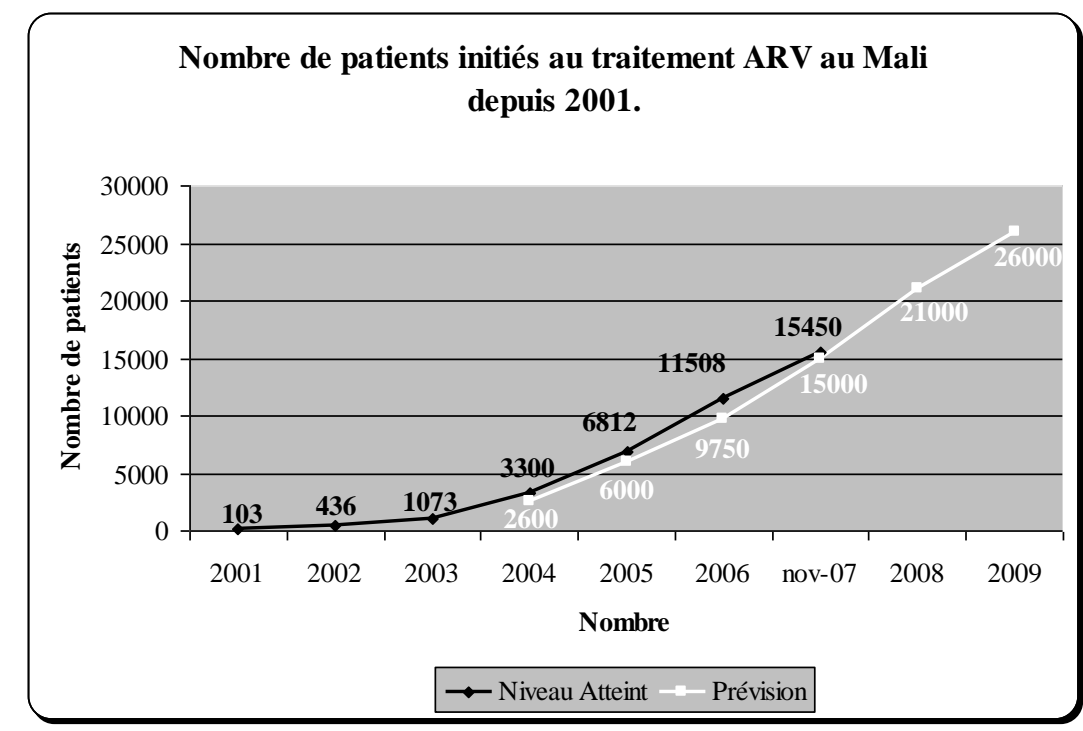

Source : M. H.A., C.S.L.S., janvier 2008.

Certains acteurs de terrain développent leurs actions sur un même territoire sans connaissances ni contacts avec les acteurs de santé travaillant au quotidien auprès des populations. Ainsi à Banconi, le Dr M. nous rapporte le cas d'une O.N.G. ayant travaillé sur le quartier dans le domaine de la prévention primaire sur le VIH/SIDA sans jamais rencontrer le personnel du centre de santé communautaire (CSCOM), que ce soit dans l'élaboration ou dans la mise en œuvre de son programme de sensibilisation alors que ce centre de santé est un organe essentiel du point de vue sanitaire et social au sein de ce quartier. La forte fréquentation de ce centre par les habitants de Banconi, la venue d'habitants provenant d'aires de santé autre que celle de Banconi (quelque fois même de villes à l'extérieur de Bamako) et sa reconnaissance internationale en témoignent.

Par ailleurs certaines ONG éprouvent des difficultés à coordonner leurs actions sur un même territoire, chacune évoluant dans «un cercle fermé » quand elles ne s'ignorent pas purement et simplement. Lors d'entretiens auprès de deux ONG maliennes en octobre 2008 rencontrées à quelques jours d'intervalle, nous avons pu noter que les deux organisations mettent en place des actions de sensibilisation dans les mêmes zones d'intervention, sans se connaître, ce qui montre encore une fois le manque de cohérence sur le terrain.

Parfois au sein d'une même organisation, les zones d'intervention sont dispersées et de profils hétérogènes. Cela entraîne non seulement des coûts élevés dans le suivi des activités, mais également une perte de performance des animateurs qui, évoluant dans des milieux disparates, doivent s'adapter et moduler les messages destinés aux populations.

Cette diversité de terrain parfois difficilement compréhensible s'explique en fait par «la logique des bailleurs de fonds » qui exigent une visibilité claire la destination de leurs fonds et la possibilité d'une évaluation individuelle de chaque intervention. Une adaptation aux contextes micro-locaux serait souhaitable.

Par ailleurs, comme nous l'avons évoqué précédemment, les messages de prévention proviennent essentiellement des grandes institutions internationales alors que leur reconnaissance par les populations destinataires des messages est relativement faible. Il en résulte un manque de crédibilité des acteurs internationaux. De plus ces messages sont essentiellement des campagnes de masses soit lors d'évènements ponctuels (ex. journée nationale de lutte contre le SIDA) ou par des canaux à large écoute (radio, télé...) mais très 
peu au sein même des quartiers alors qu'il est reconnu dans la littérature que cela est un gage de réussite.

Notre enquête montre que certains acteurs pouvant être d'un grand apport dans la lutte contre le SIDA sont peu utilisés. Ainsi le personnel de santé de première ligne est très peu sollicité. Il n'existe pratiquement pas de politique cohérente vis-à-vis de ceux qui sont pourtant dans bien des cas le premier interlocuteur des populations en ce qui concerne leur santé.

Or, notre enquête montre que la population, notamment à Banconi, ferait confiance aux agents de santé si la sensibilisation venait d'eux (Tableau 8).

Une décentralisation est donc souhaitable. Celle-ci est à l'œuvre dans le domaine curatif mais très peu en ce qui a trait à la prévention.

\section{Discussion et conclusion}

A la lecture de ces constats, on peut s'interroger sur le sens à donner aux chiffres de l'enquête démographique de santé au Mali qui annoncent une baisse du taux de prévalence du SIDA.

«Pour nous, les chiffres locaux ne cessent d'augmenter alors que les chiffres nationaux baissent, c'est troublant! (M. M., ONG Djekafo, entretien 2/10/2008)

Pour M. Traoré, anthropologue à Bamako, les données E.D.S.M. ont une "portée trop générale et parfois imprécise pour une utilisation "micro" dans une communauté restreinte».

Le discours des acteurs de terrain a néanmoins évolué. Certains d'entre eux rencontrés une première fois en 2007 et une seconde fois en 2008 après publication des résultats de l'enquête de santé ont modéré leurs critiques envers les messages de prévention.

Ainsi le responsable d'une des principales ONG nationales affirmait en 2007 «les choses n'ont pas changé, surtout dans les comportements malgré tous les efforts. [...] Tout ce que je vois, tout ce que j'entends »(M. D., ONG PSI Mali, entretien 26/01/2007). Il reconnaît aujourd'hui des progrès (timides cependant) « dans le changement des comportements ».

Il convient donc de faire une distinction entre l'échelle nationale et les multiples échelles locales et micro-locales. En d'autres termes, le progrès général masque d'importantes disparités que la forme même des données nationales de santé ne permet pas d'analyser. L'EDSM n'est pas spatialement stratifiée. Il n'est pas possible d'obtenir des données fiables à l'échelle des quartiers et l'échantillonnage même de l'enquête est sujet à caution dans certains territoires où le nombre d'individus enquêtés est insuffisamment représentatif.

Si les résultats que nous avons présentés dans ce texte ne sont que partiels puisque basés sur une exploitation incomplète de notre enquête de terrain, ils démontrent cependant la pertinence d'une lecture à l'échelle micro-locale des disparités contextuelles de perception des messages de prévention primaire et des changements de comportement face à un risque tel que la contamination par le VIH/SIDA. Dans un futur proche, nous pourrons exploiter la deuxième phase du travail entrepris et adjoindre à ces constats préliminaires d'autres indicateurs qui permettront de rendre plus précisément compte des effets de lieu dans la géographie sociale de la ville de Bamako. La question du genre, entre autres, sera essentielle à développer. "Une analyse fine du fonctionnement de la société malienne montre que les grandes décisions sont toujours prises par les hommes, en tout cas pas sans leurs avis. Par exemple, l'excision, pratique essentiellement féminine a été abandonnée dans certaines régions au Mali et ce sont les hommes qui lui ont donné le coup de grâce! » (TRAORE L.T., 2006)

Il ressort de notre enquête que l'opinion généralement répandue au Mali est que les femmes n'ont que peu de «pouvoirs » dans le domaine de la sexualité, qu'elles n'agissent que par procuration la décision finale revenant généralement à l'homme par exemple dans le choix 
ou non de recourir au port du préservatif. Cela ne signifie cependant pas que le taux de contamination soit plus élevé chez les femmes que chez les hommes, celles-ci étant en effet plus nombreuses à se faire dépister de façon volontaire et par le biais des consultations prénatales. Il s'agit là, si besoin était, d'une autre illustration du décalage existant entre une posture théorique face au risque et des comportements observés dans la réalité.

\section{Bibliographie :}

ASHFORTH A. (2002), Quand le SIDA est sorcellerie: Un défi pour la démocratie sudafricaine, Revue de Critique internationale, Numéro 14, pages 119-141

BALIQUE H., OUATTARA O., IKNANE A. Ag., (2001), Dix ans d'expérience des centres de santé communautaire au Mali, Santé publique, volume 13, no 1, pp. 35-48

BECKER C., DOZON J-P., OBBO C., TOURE M. (1998), Vivre et penser le SIDA, Karthala, 712 pages

BOILEAU C. (2007), Atelier de restitution de l'étude "déterminants des comportements sexuels à risque pour le VIH/SIDA chez les jeunes hommes et femmes de Bamako », I.N.R.S.P. Bamako, 31 pages

CAMPBELL C., MACPHAIL C. (2002), Peer education, gender and the development of critical consciousness: participatory HIV prevention, Social Science and Medicine, Volume 55, pages 331-345

MINISTERE DE LA SANTE (2007), Enquête Démographique de Santé au Mali E.D.S.M. IV, Direction Nationale de la Statistique et de l'Informatique, Cellule de Planification et de Statistique, Ministère de l'Économie, de l'Industrie et du Commerce - Bamako, 535 pages

FARMER P. (2006), Fléaux contemporains : des infections et des inégalités, Anthropos, 471 pages

KEARNS R.A. AND MOON G., 2002, From medical to health geography: theory, novelty and place in a decade of change

. Progress in Human Geography 26, pp 587-607

KEARNS R.A. AND MOON G., 2007, "A la recherche d'une nouvelle géographie de la santé" in FLEURET S., THOUEZ J.P. (dir) Géographie de la Santé, un panorama, Economica-Anthropos, pp 11-25

MAPPA S. (2005), Le savoir occidental au défi des cultures africaines: former pour changer ?, Karthala, 368 pages

MINISTERE MALIEN DE LA SANTE (2006), Enquête intégrée sur la prévalence des IST/VIH et les comportements en matière d'IST (ISBS), Ministère de la Santé, USAID, CDC, 83 pages

ONUSIDA (2008), Réponses à « Réévaluer la prévention du VIH », OnuSIDA Collection Reportages, 4 pages

ONUSIDA (1999), Sexual behavioural change for HIV: Where have theories taken us?, OnuSIDA, rapport, 60 pages

PARKHURST J.O., LUSH L. (2004), The political environment of HIV: lessons from a comparison of Uganda and South Africa, Social Science and Medicine, Volume 59, pages 1913-1924

PIOT P., (2008) Coming to terms with complexity: a call to action for HIV prevention, The Lancet, 372, pages 845-859

SCHAALMA H.P., KOK G., ABRAHAM C. Et al. (2002), L'éducation des jeunes en matière de VIH: efficacité des interventions, élaboration des éléments de programmes et travaux de recherches futures, Perspectives, Volume 32, Numéro 2, 28 pages

SINOU A. (1987), Habiter à Bamako et à Saint Louis du Sénégal, famille et résidence dans les villes africaines, L'Harmattan, 43 pages 
TRAORE L.T., (2006), L'excision au Mali : les approches de la lutte et leurs limites, Université F.L.A.S.H. de Bamako, 12 pages

UNGASS (2008), Rapport national, 74 pages, publié en ligne : http://data.unaids.org/pub/Report/2008/mali_2008_country_progress_report_fr.pdf

WAGBATSOMA VA., OKOJIE OH. (2006), Knowledge of HIV/AIDS and sexual practices among adolescents in Bénin city, Nigéria, African Journal of Reproductive Health, Volume 10, Numéro 3, pages $76-83$ 